\title{
Neuro-biological inferences into the depressive disorders of patients with chronic hepatitis $C$ treated with interferon and ribavirin
}

\author{
Dan Hurezeanu1*, Livia Dragonu', Daniela Ristea², Carmen Canciovici², Doina Ene ${ }^{2}$, Maria Balan², Madalina Sandu², \\ Dorel Cernea ${ }^{2}$ \\ From The 10th Edition of the Scientific Days of the National Institute for Infectious Diseases "Prof Dr Matei \\ Bals" \\ Bucharest, Romania. 15-17 October 2014
}

\section{Background}

This work is intended to establish a relation between two diseases, medical and psychiatric, both of them considered public health issues - chronic hepatitis $\mathrm{C}$ and depression. Interferon has been a great progress for the chronic hepatitis $C$ patients' therapy, but the treatment is encumbered by the occurrence of depression on $3-57 \%$ of patients. The purpose of this work is to assess the percent of the depressive disorder on chronic hepatitis $\mathrm{C}$ patients treated with interferon and ribavirin, as well as the monitoring of the related risk factors, upon depression occurrence and the evolution during anti-depressive treatment.

\section{Methods}

The retrospective study has been performed during the period of January 2011-January 2014 on a group of 64 patients with chronic hepatitis $C$, receiving combined antiviral treatment (interferon and ribavirin), aged between 25-64 years, observing the criteria of inclusion into treatment. The moment of psychiatric examination request has been recorded, $3.1 \%$ needing psychiatric examination before starting the therapy, $28.1 \%$ having symptoms which occurred during treatment.

\section{Results}

The psychiatric examination has revealed as speeding causes of the depressive disorder the loss of social support for $39.5 \%$, the biologic syndrome for $23 \%$, and the loss of family support for $15.4 \%$, and the depressive history for only $5.5 \%$. The percent of the depressive patients after the starting moment of the antiviral therapy has emphasized the occurrence of the depressive disorder on $83 \%$ of patients during the first 0-12 weeks. The maximum incidence of cases has been recorded on patients with ages between 20-45 years. The cases distribution has been favorable to patients from urban area -15 cases, versus 3 cases from rural area.

\section{Conclusion}

The antiviral therapy for chronic hepatitis $C$ has determined the occurrence of different levels of depression for $28.1 \%$ of the patients being studied. The anti-depressive treatment associated with the antiviral one has been efficient, decreasing the abandon rate of the antiviral therapy.

\section{Authors' details \\ "University of Medicine and Pharmacy Craiova, Romania. "Victor Babeş" Clinical Hospital of Infectious Diseases and Pneumology, Craiova, Romania.}

Published: 15 October 2014

doi:10.1186/1471-2334-14-S7-P7

Cite this article as: Hurezeanu et al:: Neuro-biological inferences into the depressive disorders of patients with chronic hepatitis $C$ treated with interferon and ribavirin. BMC Infectious Diseases 2014 14(Suppl 7):P7.

\footnotetext{
* Correspondence: dan_hurezeanu@yahoo.com

'University of Medicine and Pharmacy Craiova, Romania

Full list of author information is available at the end of the article
} 